\title{
A generalization of Darbo's fixed point and common solutions of equations in Banach spaces
}

\section{Ahmed Hajji}

"Correspondence: hajid2@yahoo.fr Laboratory of Mathematics, Computing and Application, Department of Mathematics, Faculty of Sciences, Mohammed V Agdal University, P.O. Box 1014 Rabat, Morocco

\begin{abstract}
In this paper, we present common fixed point theorems for commuting operators which generalize Darbo's and Sadovski's fixed point theorems. As example and application, we study the existence of common solutions of equations in Banach spaces using the measure of noncompactness.
\end{abstract}

MSC: $47 \mathrm{H} 10 ; 47 \mathrm{H} 09$

Keywords: fixed point; measure of noncompactness

\section{Introduction}

In 1955, Darbo [1] proved the fixed point property for $\alpha$-set contraction (i.e., $\alpha(S(A)) \leq$ $k \alpha(A)$ with $k \in] 0,1[)$ on a closed, bounded and convex subset of Banach spaces. Since then many interesting works have appeared. For example, in 1967, Sadovski [2] proved the fixed point property for condensing functions (i.e., $\alpha(S(A))<\alpha(A)$ with $\alpha(A) \neq 0)$ on closed, bounded and convex subset of Banach spaces. It should be noted that any $\alpha$-set contraction is a condensing function, but the converse is not true (see [3, p.160]). In 2007, we have proved in [4] the existence of a common fixed point for commuting mappings satisfying

$$
\begin{aligned}
& \alpha(S(A)) \leq k \sup _{i \in I}\left(\alpha\left(T_{i}(A)\right)\right), \\
& \alpha(S(A))<\sup _{i \in I}\left(\alpha\left(T_{i}(A), \alpha(A)\right)\right),
\end{aligned}
$$

where $\alpha$ is the measure of noncompactness on a closed, bounded and convex subset $\Omega$ of a locally convex space $X$, and $T_{i}$ and $S$ are continuous functions from $\Omega$ to $\Omega$ with $T_{i}$ and, in addition, are affine or linear. Furthermore, if for every $i \in I$, the $T_{i}$ in (1) and (2) are equal to the identity function, then we obtain in particular Darbo's (see [1]) as well as Sadovski's fixed point theorems (see [2]), which are used to study the existence of solutions of one equation.

The aim of this paper is to prove the existence of a common fixed point of the operators $T$ and $S$ satisfying

$$
\begin{aligned}
& \alpha(S(T(A))) \leq k \alpha(A), \\
& \alpha(S T(A))<\alpha(A),
\end{aligned}
$$

( 2013 Haiji; licensee Springer. This is an Open Access article distributed under the terms of the Creative Commons Attribution License (http://creativecommons.org/licenses/by/2.0), which permits unrestricted use, distribution, and reproduction in any medium, provided the original work is properly cited. 
where $T$ is affine, $S$ and $T$ are continuous functions, and $T$ commutes with $S$. As application, we study the existence of common solutions of the following equations:

$$
\begin{aligned}
& x(t)=f\left(t, T_{1} x(t)\right), \\
& x(t)=f(t, x(t)), \\
& x(t)=T_{2} x(t), \\
& x(t)=\lambda T_{2} x(t)+(1-\lambda) f\left(t, T_{1} x(t)\right), \quad \forall \lambda \in[0,1],
\end{aligned}
$$

under appropriate assumptions on functions $f, T_{1}$, and $T_{2}$.

\section{Preliminaries}

We begin by recalling some needed definitions and results. Let $(X,\|\|)$ be a Banach space and denote by $\mathcal{B}$ the family of all bounded subsets of $X$.

Definition 2.1 The function $\alpha: \mathcal{B} \rightarrow \mathbb{R}_{+}$defined, for every $B \in \mathcal{B}$, by

$$
\alpha(B)=\inf \left\{d>0: B \text { is the finite union of subsets } B_{i} \text { with diameter } \delta\left(B_{i}\right) \leq d\right\},
$$

is called the measure of noncompactness in $X$.

The measure of noncompactness $\alpha$ satisfies the following properties.

Proposition $2.2([1,4,5])$ Let $A, B \in \mathcal{B}$. Then

(1) For $A \subset B$, we have $\alpha(A) \leq \alpha(B)$.

(2) For any $\lambda \in \mathbb{R}$, we have $\alpha(\lambda A)=|\lambda| \alpha(A)$.

(3) $\alpha(A+B) \leq \alpha(A)+\alpha(B)$.

(4) $\alpha(\bar{A})=\alpha(A)$.

(5) $\alpha(A)=0$ if and only if $\bar{A}$ is compact.

(6) $\alpha(A \cup B) \leq \max (\alpha(A), \alpha(B))$.

(7) $\alpha(A)=\alpha(\operatorname{co}(A))$, where $\operatorname{co}(A)$ is the convex hull of $A$ in $X$.

Definition 2.3 A mapping $T$ on a convex set $\mathcal{M}$ is affine if it satisfies the identity

$$
T(k x+(1-k) y)=k T(x)+(1-k) T(y)
$$

whenever $0<k<1, x, y \in \mathcal{M}$.

Theorem 2.4 (see [4]) Let $X$ be a Hausdorff complete and locally convex space, whose topology is defined by a family of semi-norms $\mathcal{P}$. Let $\Omega$ be a convex closed bounded subset of $X, I$ be a set of index, and $\left\{T_{i}\right\}_{i \in I}$ and $S$ be two continuous functions from $\Omega$ into $\Omega$ such that:

(a) For any $i \in I, T_{i}$ commutes with $S$.

(b) For any $A \subset \Omega$

and $i \in I$, we have $T_{i}(\overline{\mathrm{co}}(A)) \subset \overline{\mathrm{co}}\left(T_{i}(A)\right)$, where $\operatorname{co}(A)$ is the convex hull of $A$ in $\Omega$.

(c) There exists $0<k<1$ such that for any $A \subset \Omega$

$$
\alpha(S(A))(p) \leq k \sup _{i \in I} \alpha\left(T_{i}(A)\right)(p), \quad p \in \mathcal{P} .
$$


Then we have:

(1) The set $\{x \in \Omega: S(x)=x\}$ is nonempty and compact.

(2) For any

$i \in I, T_{i}$ has a fixed point and $\left\{x \in \Omega: T_{i}(x)=x\right\}$ is a closed set and invariant by $S$.

(3) If $T_{i}$ is affine and $\left\{T_{i}\right\}_{i \in I}$ is a commuting family, then

$T_{i}$ and $S$ have a common fixed point and the set $\left\{x \in \Omega: T_{i}(x)=S(x)=x\right\}$ is compact.

(4) If $\left\{T_{i}\right\}_{i \in I}$ is a commuting family

and $S$ is affine, then there exists a common fixed point for the mappings $\left\{T_{i}\right\}_{i \in I}$.

Theorem 2.5 (see [4]) Let $X$ be a Hausdorff complete and locally convex space, whose topology is defined by a family of semi-norms $\mathcal{P}$. Let $\Omega$ be a convex, closed and bounded subset of $X, I$ be a given set of index, and $\left\{T_{i}\right\}_{i \in I}$, S be continuous functions from $\Omega$ into $\Omega$ such that:

(a) For every $i \in I, T_{i}$ commutes with $S$.

(b) For every $i \in I, T_{i}$ is linear.

(c) There exists $j \in I$ such that for every $A \subset \Omega$ and $p \in \mathcal{P}$, with $\alpha(A)(p) \neq 0$, we have

$$
\alpha(S(A))(p)<\sup \left(\alpha\left(T_{j}(A)(p), \alpha(A)(p)\right)\right) .
$$

Then

(1) $T_{j}$ and $S$ have a fixed point, and $\left\{x \in \Omega: T_{j}(x)=S(x)=x\right\}$ is a nonempty and compact set.

(2) If $\left\{T_{i}\right\}_{i \in I}$ is a commuting family and $S$ is affine, then there exists a common fixed point for the mappings in $\left\{T_{i}\right\}_{i \in I}$.

\section{Fixed point theorems}

It is well known that if $S T$ has a fixed point, for given operators $T$ and $S$, then $S$ and $T$ not necessarily have a fixed point or a common fixed point. Thus, it will be of interest to establish some results showing the existence of a common fixed point for $T$ and $S$ when the operator $S T$ has a fixed point. The last fact can be used to study the existence of common solutions of equations.

Theorem 3.1 Let $X$ be a Banach space and $\Omega$ be a nonempty convex, closed and bounded subset of $X$. Let $T$ and $S$ be two continuous functions from $\Omega$ into $\Omega$ such that:

(a) $T S=S T$.

(b) $T$ is affine.

(c) There exists $k \in] 0,1[$ such that for any $A \subset \Omega$ we have

$$
\alpha(S T(A)) \leq k \alpha(A) .
$$

Then the set $\{x \in \Omega: S x=T x=x\}$ is nonempty and compact.

Proof Consider the operator $H(x)=k S(T(x))+(1-k) T(x)$. It is clear that $H$ maps $\Omega$ into $\Omega$, commutes with $T$ and is continuous. Moreover, by (2) and (3) of Proposition 2.2, we have

$$
\alpha(H(A))=\alpha(k S(T(A))+(1-k) T(A)) \leq k^{2} \alpha(A)+(1-k) \alpha(T(A))
$$


for any $A \subset \Omega$. Furthermore, since $k \in] 0,1\left[\right.$ we have $k^{2}<k$ and therefore $k^{2}+1-k<k+1-$ $k=1$. Hence by making appeal to Theorem 2.4 , we conclude that $\mathcal{F}_{0}=\{x \in \Omega / H x=T x=x\}$ is nonempty and compact. Furthermore, for any $x \in \mathcal{F}_{0}$, we have

$$
H(x)=k S T(x)+(1-k) T(x)=T(x)=x \quad \Rightarrow \quad S(x)=x .
$$

Whence, $S$ and $T$ have a common fixed point. Let $\mathcal{F}=\{x \in \Omega: S x=T x=x\}$, then

$$
\alpha(S T(\mathcal{F}))=\alpha(\mathcal{F}) \leq k \alpha(\mathcal{F})
$$

which implies $\alpha(\mathcal{F})=0$. Now, since $S$ and $T$ are continuous, then $\mathcal{F}$ is compact.

Observe that Theorem 3.1 above shows the following:

(*) If the operator $S T$ has a fixed point, then $T$ and $S$ have a common fixed point.

(**) If the operator $T$ equals the identity function, then we obtain Darbo's fixed point theorem.

Theorem 3.2 Let $X$ be a Banach space and $\Omega$ be a nonempty convex, closed and bounded subset of $X$. Let $T_{1}, T_{2}$, and $S$ be continuous functions from $\Omega$ into $\Omega$ such that:

(a) $T_{1} T_{2}=T_{2} T_{1}$ and $T_{i} S=S T_{i}$ for any $i \in\{1,2\}$.

(b) $T_{1}, T_{2}$ are affine.

(c) There exists $k \in] 0,1[$ such that for any $A \subset \Omega$ we have

$$
\alpha(S(A)) \leq k \alpha(A)
$$

Then the set $\left\{x \in \Omega: S x=T_{1} x=T_{2} x=x\right\}$ is nonempty and compact.

Proof Consider the following operator $H(x)=S\left(T_{1}(x)\right)$. It is clear that $H$ maps $\Omega$ into $\Omega$, commutes with $T_{1}$, and is continuous. Moreover, we have for any $A \subset \Omega$

$$
\alpha(H(A))=\alpha\left(S\left(T_{1}(A)\right)\right) \leq k \alpha\left(T_{1}(A)\right) .
$$

Hence, by Theorem 2.4, $H$ and $T_{1}$ have a common fixed point which is a fixed point with $S$. Thus, the nonempty set $\mathcal{F}=\left\{x \in \Omega: T_{1} x=x\right\}$ is convex, closed and bounded subset of $\Omega$, for $T_{1}$ being continuous and affine. Moreover, by (a) we have $S(\mathcal{F}) \subset \mathcal{F}$ and $T_{2}(\mathcal{F}) \subset \mathcal{F}$. Therefore, for any $A \subset \mathcal{F}$, we have

$$
\alpha\left(S\left(T_{2}(A)\right)\right) \leq k \alpha\left(T_{2}(A)\right) .
$$

By the same argument as before, we consider $H_{1}(x)=S T_{2}(x)$ for $x \in \mathcal{F}$. It follows that the set $\left\{x \in \Omega: S x=T_{1} x=T_{2} x=x\right\}$ is nonempty and compact.

Theorem 3.2 can be used to show that any affine operator $T$ that commutes with $S$ and satisfies Darbo's condition [1] has a common fixed point with $S$.

Theorem 3.3 Let $X$ be a Banach space, $\Omega$ be a nonempty convex, closed and bounded subset of $X, T$ and $S$ be continuous functions from $\Omega$ into $\Omega$ such that: 
(a) T commutes with $S$.

(b) $T$ is linear.

(c) For any $A \subset \Omega$ with $\alpha(A) \neq 0$, we have

$$
\alpha(S(T(A)))<\alpha(A)
$$

Then the set $\{x \in \Omega: S x=T x=x\}$ is nonempty.

Proof Let $k \in] 0,1[$ and consider the operator

$$
H(x)=k S T(x)+(1-k) T x
$$

for any $x \in \Omega$. It is clear that $H$ maps $\Omega$ into $\Omega$, commutes with $T$, and is continuous. Moreover, for any $A \subset \Omega$ such that $\alpha(A) \neq 0$, we have

$$
\begin{aligned}
\alpha(H(A)) & =\alpha(k S T(A)+(1-k) T(A)) \\
& \leq k \alpha(S T(A))+(1-k) \alpha T(A)<k \alpha(A)+(1-k) \alpha T(A) .
\end{aligned}
$$

Hence, by Theorem 2.5, $H$ and $T$ have a common fixed point which is also a fixed point of $S$.

As a consequence of Theorem 3.3 above, one can recover Sadovski's fixed point theorem when $T$ is equal to the identity function.

\section{Example and application}

\subsection{Example}

Let $L^{1}\left(\mathbb{R}_{+}\right)$be the space of Lebesgue integrable functions on the measurable subset $\mathbb{R}_{+}$of $\mathbb{R}$ with the standard norm

$$
\|x\|=\int_{0}^{+\infty}|x(t)| d t
$$

Let $k \in L^{1}\left(\mathbb{R}_{+}\right)$defined by

$$
k(t)= \begin{cases}e^{-t} & \text { for } t \in[0,1] \\ 0 & \text { for } t>1\end{cases}
$$

Therefore, we can see that for any $A>0$ and for all $t_{1}, t_{2} \in \mathbb{R}_{+}$, the following condition is satisfied:

$$
t_{1}<t_{2} \Rightarrow \int_{0}^{A} k\left(t_{2}-s\right) d s \leq \int_{0}^{A} k\left(t_{1}-s\right) d s .
$$

Then using Krzyz's theorem (see [6]), the linear operator

$$
(K x)(t)=\int_{0}^{\infty} k(t-s) x(s) d s
$$


transforms the set of non-increasing functions from $L^{1}\left(\mathbb{R}_{+}\right)$into itself. Furthermore, we can see that the norm of the convolution operator $K$ satisfies

$$
\|K\| \leq 1-e^{-1} .
$$

The Hausdorff measure of noncompactness on the Banach space $E$, noted by $\chi(A)$ (see $[5,7])$, is defined as

$$
\chi(A)=\inf \left\{r>0 \text { : there exists a finite subset } Y \subset E \text { such that } A \subset Y+B_{r}\right\},
$$

where $B_{r}$ is a closed ball in $E$ centered at zero and of radius $r$.

Note that there is another measure $\gamma$ on the space $L^{1}\left(\mathbb{R}_{+}\right)$[8]. Indeed, for any $\epsilon>0$, let

$$
C(X)=\lim _{\epsilon \rightarrow 0} \sup _{x \in X} \sup \left\{\int_{D}|x(t)| d t: D \subset \mathbb{R}_{+}, \operatorname{meas}(D) \leq \epsilon\right\},
$$

where meas $(D)$ denotes the Lebesgue measure of the subset $D$ (see $[7,8]$ ), and

$$
d(x)=\lim _{T \rightarrow \infty} \sup \left\{\int_{T}^{\infty}|x(t)| d t: x \in X\right\} .
$$

We have

$$
\gamma(X)=C(X)+d(X)
$$

Then we have the following theorem.

Theorem 4.1 (see [8]) Let $X$ be a nonempty, bounded and compact in measure subset of $L^{1}\left(\mathbb{R}_{+}\right)$. Then

$$
\chi(X) \leq \gamma(X) \leq 2 \chi(X)
$$

Now, let us consider the following operator defined by

$$
S(x)(t)=b K x(\varphi(t))+e^{-t}=b \int_{0}^{\infty} k(t-s) x(\varphi(s)) d s+e^{-t}
$$

for any $x \in L^{1}\left(\mathbb{R}_{+}\right)$, where $b>0$ and the function $\varphi: \mathbb{R}_{+} \rightarrow \mathbb{R}_{+}$is increasing and absolutely continuous such that $\varphi^{\prime}(t) \geq B$ for some constant $B>0$ and for almost all $t \in \mathbb{R}_{+}$.

Using the same argument as in [7], we can show that for any $x \in L^{1}\left(\mathbb{R}_{+}\right)$, we have

$$
\|S x\|=\int_{0}^{\infty}|S x(t)| d t \leq \int_{0}^{\infty} e^{-t} d t+\frac{b\|K\|}{B}\|x\|=1+\frac{b\|K\|}{B}\|x\| .
$$

Hence, for $\|x\|<r$, we have

$$
\|S x\|<1+\frac{b\|K\|}{B} r \leq 1+\frac{b\left(1-e^{-1}\right)}{B} r,
$$


and if we take

$$
1+\frac{b\left(1-e^{-1}\right)}{B} r=r
$$

then

$$
r=\frac{1}{1-\frac{b}{B}\left(1-e^{-1}\right)} .
$$

Moreover,

$$
\|K\| \leq 1-e^{-1} .
$$

This implies that $S$ and $K$ map the ball $B_{r}$ into $B_{r}$, where $r=\frac{1}{1-\frac{b}{B}\left(1-e^{-1}\right)}$ with $b<\frac{B e}{e-1}$.

Further, let $Q_{r}$ be the subset of $B_{r}$ consisting of all functions that are a.e. positive and nonincreasing on $\mathbb{R}_{+}$, which is a compact in measure (see [9]), bounded, closed and convex subset of $L^{1}\left(\mathbb{R}_{+}\right)$. Therefore, $K$ and $S$ map $Q_{r}$ into $Q_{r}$ (see [7, p.454]). Whence, for any $A \in Q_{r}$, we have

$$
\gamma(S(A)) \leq \frac{b\|K\|}{B} \gamma(A) \leq \frac{b}{B}\left(1-e^{-1}\right) \gamma(A) .
$$

Next, by making use of Theorem 4.1, we get

$$
\chi(S(A)) \leq 2 \frac{b\|K\|}{B} \chi(A) \leq 2 \frac{b}{B}\left(1-e^{-1}\right) \chi(A) .
$$

This implies that $S$ is a $\chi$-contraction with the constant $2 \frac{b\|K\|}{B}$ or $2 \frac{b}{B}\left(1-e^{-1}\right)$. If we take $b \in] \frac{1}{2} \frac{B e}{e-1}, \frac{B e}{e-1}\left[\right.$, then the constant $2 \frac{b}{B}\left(1-e^{-1}\right)>1$. Hence, neither Darbo's fixed point nor Sadovski's fixed point theorem are applied to the operator $S$. On the other hand, we have $S K=K S$, indeed the function $e^{-t}$ for any $t \in \mathbb{R}_{+}$is a fixed point of $K$ (see [4]), $S K$ maps $Q_{r}$ into $Q_{r}$ and

$$
\gamma(S K(A)) \leq \frac{b\|K\|^{2}}{B} \gamma(A) \leq \frac{b}{B}\left(1-e^{-1}\right)^{2} \gamma(A) .
$$

Thus,

$$
\chi(S K(A)) \leq 2 \frac{b\|K\|^{2}}{B} \chi(A) \leq 2 \frac{b}{B}\left(1-e^{-1}\right)^{2} \chi(A),
$$

which implies that $S K$ is a $\chi$-contraction with the constant $2 \frac{b}{B}\left(1-e^{-1}\right)^{2}<1$. Now, by Theorem 3.1, $S$ and $K$ have a common fixed point $x_{0} \in Q_{r}$ satisfying

$$
b x_{0}(\varphi(t))+e^{-t}=x_{0}(t)=\int_{0}^{+\infty} k(t-s) x_{0}(s) d s .
$$

This gives rise to a solution of the following equation:

$$
x(t)=b \int_{0}^{\infty} k(t-s) x(\varphi(s)) d s+e^{-t} .
$$




\subsection{Common solutions of equations in Banach spaces}

Let $(X,\|\|)$ be a Banach space and $B$ be a convex, closed and bounded subset of $X$. Denote by $C([0, b], B)$ the space of all continuous functions from $[0, b] ; b>0$, into $B$ endowed with the norm

$$
\|x\|_{\infty}=\sup _{t \in[0, b]}\|x(t)\| .
$$

Assume that

(a) for given fixed $f:[0, b] \times B \rightarrow B$, there exists $k \in] 0,1[$ such that

$$
\|f(t, x)-f(t, y)\| \leq k\|x-y\|
$$

for all $x, y \in B, t \in[0, b]$;

(b) $T_{i}: B \rightarrow B$ are linear continuous, satisfying $T_{i}(f(t, x))=f\left(t, T_{i}(x)\right)$ for any $(t, x) \in[0, b] \times B$ and $\forall i \in\{1,2\}$.

Theorem 4.2 Under hypotheses (a) and (b), equations (5), (6), (7), and (8) have at least one common solution in $C([0, b], B)$.

Proof First, it is clear that $C([0, b], B)$ is a closed, bounded and convex subset of $C([0, b], X)$. On the other hand, by considering $S x(t):=f(t, x(t))$, for $x \in C([0, b], B)$, we have

$$
\|S x(t)-S y(t)\| \leq k\|x(t)-y(t)\| \leq k\|x-y\|_{\infty} .
$$

This implies that

$$
\|S x-S y\|_{\infty} \leq k\|x-y\|_{\infty}
$$

for any $x, y \in C([0, b], B)$. Furthermore, since any contraction with the constant $k$ is an $\alpha$-contraction with the same constant $k$ ( $\alpha$ is the measure of noncompactness in $C([0, b], B))$, then

$$
\alpha(S(A)) \leq k \alpha(A) \quad \text { for any } A \in C([0, b], B) .
$$

Finally, since $S$ and $T_{i}$ commute, we conclude from Theorem 3.2 that $T_{1}, T_{2}$, and $S$ have a common fixed point. Therefore, equations (5), (6), (7), and (8) have at least one common solution in $C([0, b], B)$.

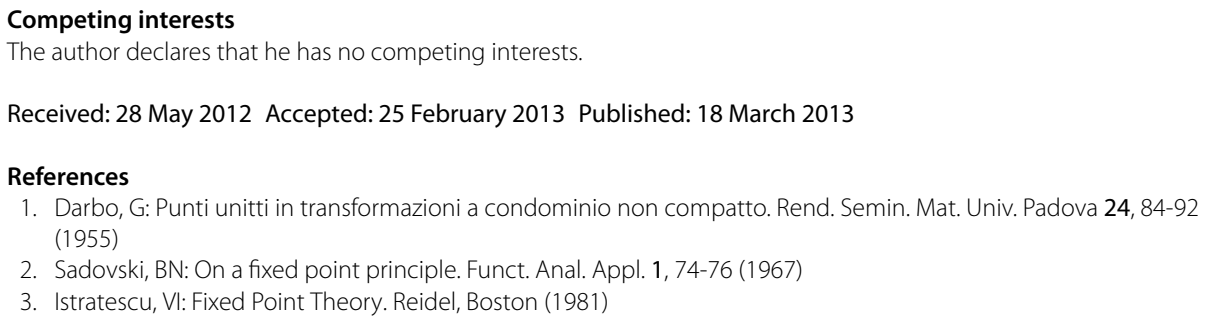


4. Hajji, A, Hanebaly, E: Commuting mappings and $\alpha$-compact type fixed point theorems in locally convex spaces. Int J. Math. Anal. 1(14), 661-680 (2007)

5. Banas, J, Goebel, K: Measure of Noncompactness in Banach Spaces. Lect. Notes in Math., vol. 60. Dekker, Now York (1980)

6. Krzyz, J: On monotonicity-preserving transformations. Ann. Univ. Mariae Curie-SkT̄odowska, Sect. A, 6, 91-111 (1952)

7. El-Sayed, WG: Nonlinear functional integral equations of convolution type. Port. Math. 54(4), 449-456 (1997)

8. Banas, J, El-Sayed, WG: Measure of noncompactness and solvability of an integral equation in class of functions of locally bounded variation. J. Math. Anal. Appl. 167, 133-151 (1992)

9. Dunford, N, Schwartz, JT: Linear Operators I. Interscience, Leyden (1963)

doi:10.1186/1687-1812-2013-62

Cite this article as: Hajji: A generalization of Darbo's fixed point and common solutions of equations in Banach spaces. Fixed Point Theory and Applications 2013 2013:62.

Submit your manuscript to a SpringerOpen ${ }^{\circ}$ journal and benefit from:

- Convenient online submission

Rigorous peer review

- Immediate publication on acceptance

- Open access: articles freely available online

- High visibility within the field

- Retaining the copyright to your article 